\title{
NOTE ON HELIUMLIKE SILICON AND SULFUR LINES OBSERVED IN THE X-RAY SPECTRA OF SOLAR FLARES
}

\author{
(Research Note-Erratum)
}

\author{
G. A. DOSCHEK and J. F. MEEKINS \\ E. O. Hulburt Center for Space Research, Naval Research Laboratory, \\ Washington, D.C. 20390, U.S.A.
}

(Received 30 October, 1972)

We have reported on the time behavior and relative intensities of the resonance $\left(1 s^{2}\right.$ $\left.{ }^{1} S-1 s 2 p{ }^{1} P\right)$, intercombination $\left(1 s^{2}{ }^{1} S-1 s 2 p{ }^{3} P\right)$, and forbidden $\left(1 s^{2}{ }^{1} S-1 s 2 s{ }^{3} S\right)$ lines of the heliumlike ions of silicon and sulfur as observed in solar flare X-ray spectra obtained by OSO-4 (Doschek and Meekins, 1970). Figure 2 of the above paper shows that near 2159 UT and 2216 UT the intensity of the forbidden line of Si XIII exceeds the intensity of the resonance line. Figure 3 shows a similar result for two scans of $\mathrm{S} x \mathrm{xv}$. Conversely, near $2146 \mathrm{UT}$, the $\mathrm{S}$ xv forbidden line appears to be absent in the spectrum. At the time of publication, these results appeared somewhat surprising but were nevertheless accepted because the lines were observed during flares, and the intensity behavior of the forbidden lines was assumed to be caused by non-steady state conditions in the flare plasma.

Since 1970, however, considerably more data for these lines have become availablefrom OSO-5 and OSO-6, e.g., Neupert (1971), and Doschek (1972). These data show rather conclusively that the intensities of the forbidden lines of the heliumlike ions observed in flare spectra never equal or exceed the intensities of the resonance lines. Furthermore, the forbidden line of $\mathrm{S} \mathrm{XV}$ is never observed to be as weak as in the $2146 \mathrm{UT}$ scan described above. These observations are also in agreement with the theory of the formation of these lines, e.g., see Freeman et al. (1971).

We have concluded that in reducing the OSO-4 heliumlike silicon data, we underestimated the amount of silicon in the mica window of the OSO-4 detector. The silicon in the mica window produces an absorption edge in the data near $6.74 \AA$; the forbidden line falls on the long-wavelength side of this edge, while the other two lines are on the short-wavelength side of the edge. The difficulty with the OSO-4 S xV data is not yet apparent; we have simply never obtained similar data with the OSO-6 instrument, which has observed many more, and larger events than we recorded from OSO-4.

Consequently, the OSO-4 data published in Doschek and Meekins (1970) should not be used in theoretical considerations until the instrumental problems are resolved. The OSO-5 and OSO- 6 results for these lines obtained by the Goddard and NRL groups may be found in a variety of publications, e.g., see the references given above.

We are currently working on instrumental corrections for our OSO-6 instruments, 
as well as improved parameters for the OSO-4 instrument. These will be published in a subsequent communication.

\section{References}

Doschek, G. A.: 1972, Space Sci. Rev. 13, 765.

Doschek, G. A. and Meekins, J. F.: 1970, Solar Phys. 13, 220.

Freeman, F. F., Gabriel, A. H., Jones, B. B., and Jordan, C.: 1971, Phil. Trans. Roy. Soc. London A270, 127.

Neupert, W. M.: 1971, Phil. Trans. Roy. Soc. London A270, 143. 